\title{
$k$-TO-1 FUNCTIONS BETWEEN GRAPHS WITH FINITELY MANY DISCONTINUITIES
}

\author{
JO HEATH
}

(Communicated by Dennis Burke)

\begin{abstract}
This paper classifies which pairs of graphs $G$ and $H$ and which integers $k \geq 2$ allow $k$-to- 1 functions from $G$ onto $H$ with only finitely many discontinuities.
\end{abstract}

1. Introduction. It has long been known that there are no 2-to-1 continuous functions defined on graphs with odd Euler number [3], nor are there $k$-to-1 continuous functions onto any dendrite [4], and hence any acyclic graph, for $k>1$. There are many other results on continuous $k$-to-1 maps; see $[\mathbf{1}, \mathbf{2}, \mathbf{5}, \mathbf{6}, \mathbf{1 2}-\mathbf{1 5}]$.

More recently, studies have been made to determine which spaces allow $k$-to- 1 functions (to them or from them) with only a finite number of discontinuities. For instance, odd-Euler graphs do not support 2-to-1 functions with only finitely many discontinuities [7] and for $k>2$, Kellum and Katsuura proved that every $k$-to-1 function from $[0,1]$ onto $[0,1]$ has infinitely many discontinuities $[\mathbf{1 0}]$. This latter result was generalized in [9] to functions from continua onto dendrites. See also [8, 11].

This paper answers the question: given an integer $k>1$, for which finite graphs (1-complexes) $G$ and $H$ does there exist a $k$-to-1 function from $G$ onto $H$ with only finitely many discontinuities? The principal feature of a dendrite that prohibits its being the image of a $k$-to- 1 function is its lack of simple closed curves, and for graphs, the number of simple closed curves in $G$ and in $H$ completely determines the answer to the above question.

There are a lot more questions about $k$-to- 1 functions, discontinuous or continuous, than there are answers, and here are a few:

QUESTION 1 (R. LEVY [12]). Is a circle the only 2-to-1 continuous image of $[0,1) ?$

QUESTION 2. Can the results of this paper be generalized to 2-dimensional finite complexes?

QUESTION 3. For which $k$ and for which continua $X$ and $Y$ are uncountably many discontinuities required of any $k$-to-1 function from $X$ onto $Y$ ?

2. Definitions and Lemma 0. A function with only finitely many discontinuities will be called a $F D$-function. The continuous case is not excepted.

If $G$ is a finite graph whose $n_{1}$ endpoints and junction points separate $G$ into $m_{1}$ disjoint open arcs, and if another set in $G$ with $n$ points separates $G$ into $m$

Received by the editors May 1, 1987. Presented to the Spring Topology Conference, Gainesville, Florida, April 1988.

1980 Mathematics Subject Classification (1985 Revision). Primary 05C10, 26A15, 54C10.

Key words and phrases. $k$-to-1 function, 2-to-1 function, graph. 
disjoint open arcs, then the $n$ points contain the $n_{1}$ points plus a few from the $m_{1}$ arcs, and $m-n=m_{1}-n_{1}$. Thus the following is well defined:

A graph $G$ is $j$-multicyclic if there are $n$ points in $G$ whose complement in $G$ is the union of $m$ disjoint open arcs and $j=m-n+1>0$. If $j=1, G$ is called unicyclic.

Note that if $G$ is embedded in the plane, then $G$ is $j$-multicyclic iff $G$ has $j$ bounded complementary domains.

The following notion drastically reduces the number of cases. For instance, it permits every acyclic graph to be treated as the interval $[0,1]$.

Suppose the space $X$ is the union of $n$ disjoint subsets, $X=X_{1}+X_{2}+\cdots+X_{n}$, and $Y$ is also the union of $n$ disjoint subsets $Y=Y_{1}+Y_{2}+\cdots+Y_{n}$. Suppose further that there is a function $h$ from $X$ onto $Y$ whose restriction to $X_{i}$ is a homeomorphism onto $Y_{i}$, for each $i=1,2, \ldots, n$. Then $X$ is a rearrangement of $Y$ if for each function $f$ defined on $Y, f$ has finitely many discontinuities iff $f h$ (defined on $X$ ) has finitely many discontinuities.

For instance, the $\theta$ curve is a rearrangement of $(0,1)+(2,3)+(4,5)+\{6,8\}$, and so is $(0,1)$, by thinking of $(0,1)$ as $\left(0, \frac{1}{3}\right)+\left(\frac{1}{3}, \frac{2}{3}\right)+\left(\frac{2}{3}, 1\right)+\left\{\frac{1}{3}, \frac{2}{3}\right\}$. Note that rearrangement is an equivalence relation.

The next lemma will be used often without specific mention:

LEMMA 0 . If $G$ is an acyclic graph, then $[0,1]$ is a rearrangement of $G$; if $G$ is unicyclic, then $[0,1)$ is a rearrangement of $G$; and if $G$ is $j$-multicyclic with $j>1$, then the disjoint union of $j-1$ open intervals is a rearrangement of $G$.

PROOF. For all three cases, let $n$ denote the number of endpoints and junction points of $G$ plus one (to take care of $G=S^{1}$ ), let $m$ denote the number of open arcs in the complement of these $n$ points, and let $j=m-n+1$. Then $T=$ $(1,2)+(2,3)+\cdots+(m, m+1)+\{1,2, \ldots, n\}$ is a rearrangement of $G$.

Since every acyclic graph has $n=m+1,[1, m+1]$ is a rearrangement of $T$ and hence of $G$. If $G$ is unicyclic then $j=1$, so $n=m$ and $[1, m+1)$ is a rearrangement of $T$ and of $G$. Now suppose $j>1$. If $n<m$, then $m$ open intervals plus $n$ points rearrange as $m-n$ open intervals. Since $j$ is greater than $1, n=m+1-j$ is less than $m$, so that $G$ rearranges as the disjoint union of $m-(m+1-j)$ or $j-1$ open intervals.

\section{Reference facts from other papers:}

1. [7] There is no 2-to-1 function on $[0,1]$ or on $(0,1)$ with finitely many discontinuities, with Hausdorff image.

2. [9] There is no $k$-to-1 function from any continuum onto $[0,1]$ with only finitely many discontinuities, for $k>1$.

3. [11] There is no $k$-to- 1 function from $(0,1)$ onto $[0,1)$ with only finitely many discontinuities, for $k>1$.

4. [11] For every $k>1$ there is a $k$-to- 1 function from $(0,1)$ onto $(0,1)$ with only finitely many discontinuities.

5. $[10,11]$ For each $k>2$, there is a $k$-to- 1 function from $[0,1]$ onto $(0,1)$ with only finitely many discontinuities.

6. $[6,10]$ For each $k>2$, there is a $k$-to- 1 function from $[0,1]$ onto $[0,1)$ with only finitely many discontinuities. 
4. Lemmas. These lemmas will be used in the next section on the rearranged graphs.

LEMMA 1. There is no 2-to-1 function from $[0,1)$ onto the union of $t$ disjoint open intervals with only finitely many discontinuities.

ProOF. Suppose there is such a function $f$. Let $D \subseteq[0,1)$ be the set of discontinuities of $f$ plus the number 0 . Then the $t$ open intervals of the image minus the $n$ points of $f(D)$ is the union of $t+n$ disjoint open intervals, $J_{1}+\cdots+J_{t+n}$. Since $f$ is 2 -to- $1, f^{-1}(f(D))$ has $2 n$ points, $0=x_{1}<\cdots<x_{2 n}$. Let $x_{2 n+1}=1$. Since $f$ restricted to each $\left(x_{i}, x_{i+1}\right)$ for $i=1,2, \ldots, 2 n$ is continuous, it maps each into only one $J_{j}$. For each $j=1,2, \ldots, t+n$, let $h(j)$ denote the number of open intervals $\left(x_{i}, x_{i+1}\right)$ that map into $J_{j}$. Then

1. $h(1)+h(2)+\cdots+h(n+t)=2 n$ and

2. $h(1)+h(2)+\cdots+h(n+t) \geq 2 n+2 t$.

The first is true because there are $2 n$ of the $\left(x_{i}, x_{i+1}\right)$ intervals, the second is true because $h(j) \geq 2$ for each $j$ by reference fact 1 . This contradiction completes the proof. (The proof of this lemma is a variation of a proof in [10].)

LEMMA 2. There is no $k$-to-1 function, $k>1$, from the disjoint union of $m$ open intervals onto $[0,1)$ with only finitely many discontinuities.

PROOF. Suppose there is such a function $f$. Let $q$ be a positive integer such that $q m \geq k$. Then $q$ copies of $f$ take $m q$ open intervals onto $[0,1)+[1,2)+\cdots+[q-1, q)=$ $[0, q)$. This function can be extended to a $k$-to- $1 F D$-function taking the $q m$ open intervals plus $k$ new points onto $[0, q]$ by sending the new points to $q$. But $q m$ open intervals plus $k$ points can be rearranged as a continuum since $k \leq q m+1$. This contradicts reference fact 2 .

LEMMA 3. If $k>1$ and $0<m \leq k$, then there is a $k$-to-1 function from the union of $m$ disjoint open intervals onto $(0,1)$ with only finitely many discontinuities, except for the one case $m=1$ and $k=2$.

PROOF. If $m \leq k$, then $r=k-m \geq 0$. If $r \neq 1$, then there is a $(r+1)$-to-1 $F D$-function from the first open interval in the domain onto $(0,1)$ by reference fact 4. Then each of the remaining $m-1$ open intervals of the domain can be mapped 1 -to- 1 onto $(0,1)$. The union of these maps is the required $k$-to- $1 F D$-function. If $r=1$ then $m=k-1$ so the $m$ open intervals plus $k$ new points rearranges to $[0,1]$, and the image $(0,1)$ plus 1 new point is $[0,1)$. By reference fact 6 , if $k \neq 2$, there is a $k$-to- $1 F D$-function $f$ from $[0,1]$ onto $[0,1)$. (If $k=2$ then $m=k-1=1$ is the one excluded case.) Then $[0,1]$ minus the $k$ points of $f^{-1}(0)$ maps via $f$ to $(0,1)$. But $[0,1]$ minus $k$ points rearranges to $k-1$ intervals so the restricted $f$ is the required function.

LEMMA 4. If $m>k>1$, then there is no $k$-to-1 function from the union of $m$ disjoint intervals onto $(0,1)$ with only finitely many discontinuities.

PROOF. Since $m>k, m-k$ is positive. Let $q$ be an integer such that $q>$ $(k-1) /(m-k)$. Then $q m+1>k(q+1)$. Suppose there is a $k$-to-1 $F D$-function from the $m$ open intervals onto $(0,1)$. Then the union, $h$, of $q$ copies of this function maps the union of $q m$ open intervals onto the union of $q$ open intervals. But since 
$q m+1>k(q+1)$, the $q m$ open intervals plus $k(q+1)$ new points can be rearranged into a continuum $M$. Then $h$ extends to $M$ by mapping the $k(q+1)$ new points to $q+1$ new points in the range. But the new image of $q$ open intervals plus $q+1$ points rearranges as $[0,1]$ contradicting reference fact 2 .

LEMMA 5. If $k>2$ then there is a $k$-to-1 function from $[0,1)$ onto $(0,1)$ with only finitely many discontinuities.

ProOF. Let $k>2$. Let $g$ and $h$ be any 3-to- $1 F D$-functions from $[0,1]$ onto $[0,1)$ and from $(0,1)$ onto $(0,1)$ respectively. See reference facts 6 and 4 . The functions for $k=3,4$ and 5 will be painfully constructed and then all other functions are unions of these three maps.

(a) Define the 3-to-1 $F D$-function $f 3$ from $[0,2)$ onto $(0,2)$ by

$$
\begin{aligned}
f 3(x) & =1-g(x) & & \text { if } x \in[0,1] \\
& =h(x)+1 & & \text { if } x \in(1,2) .
\end{aligned}
$$

(b) Define the 4-to-1 $F D$-function $f 4$ from $[0,3)$ onto $(0,2)$ by

$$
\begin{aligned}
f 4(x) & =h(x) & & \text { if } x \in(0,1), \\
& =h(x)+1 & & \text { if } x \in(1,2), \\
& =2(x-2) & & \text { is } x \in(2,3), \\
& =1 & & \text { if } x=0,1 \text { or } 2 .
\end{aligned}
$$

(c) Define the 5-to-1 $F D$-function $f 5$ from $[0,5)$ onto $(0,3)$ by:

$$
\begin{aligned}
f 5(x) & =h(x) & & \text { if } x \in(0,1), \\
& =h(x)+1 & & \text { if } x \in(1,2), \\
& =g(x)+2 & & \text { if } x \in[2,3], \\
& =3(x-3) & & \text { if } x \in(3,4), \\
& =3(x-4) & & \text { if } x \in(4,5), \\
& =1 & & \text { if } x=0,1 \text { or } 4 .
\end{aligned}
$$

Now, to avoid getting bogged down in endpoint values, think of each $f k, k=3,4,5$, as a generic $k$-to-1 $F D$-function from a half open interval onto an open interval.

If $k>5$, write $k=3 q+4 r+5 t$ for nonnegative integers $q, r$ and $t$. Inductively, it is clear that $k=3,4,5$ can be so written and that $k$ can be if $k-3$ can be.

The domain $[0,1)$ can be rearranged as $q+r+t$ disjoint half-open intervals. Map the first $q$ of them onto $(0,1)$ via $f 3$, the next $r$ of them onto $(0,1)$ via $f 4$, and the last $t$ of them onto $(0,1)$ via $f 5$. The union of these $r+s+t$ functions is a $k$-to- 1 $F D$-function from $[0,1)$ onto $(0,1)$.

\section{Theorems and proofs.}

THEOREM 1. If $G$ is a $(m+1)$-multicyclic graph and $H$ is a $(t+1)$-multicyclic graph, then there is a $k$-to-1 function from $G$ onto $H$ with only finitely many discontinuities:

(A) iff $m \leq k t$, if $k>2$, and

(B) iff $m=2 t$, if $k=2$.

PROOF. 1. A reduction. 
Suppose $m \geq k+1$ and $t>1$. Then neither $G$ nor $H$ is unicyclic. Hence $G$ can be rearranged as the disjoint union of $m$ open intervals and $H$ as $t$ open intervals. There is a $k$-to- $1 F D$-function from $m$ open intervals onto $t$ open intervals iff there is one from $m$ open intervals plus $k$ new points onto $t$ open intervals plus 1 new point. But $m$ open intervals and $k$ points rearrange to $m-k$ open intervals, and $t$ open intervals and one point rearrange to $t-1$ open intervals. If $q$ is the largest integer such that $m^{\prime}=m-q k>0$ and $t^{\prime}=t-q>0$ then $m^{\prime} \leq k$ or $t^{\prime} \leq 1$ and there is a $F D$-function from $G$ onto $H$ iff there is one from $m^{\prime}$ open intervals onto $t^{\prime}$ open intervals. Also note that $m \leq k t$ iff $m^{\prime} \leq k t^{\prime}$ and $m=2 t$ iff $m^{\prime}=2 t^{\prime}$. Hence it can be assumed that

$$
t \leq 1 \quad \text { or } \quad m \leq k
$$

2. Proof of (A) and (B) if $G$ and $H$ are both unicyclic. $G$ can be rearranged as $[0, k)$ and $H$ can be rearranged as $[0,1)$. Define the $k$-to- $1 F D$-function $f$ from $[0, k)$ onto $[0,1)$ by $f(x)=x-\lfloor x\rfloor$.

3. Proof of (A).

$(\leftarrow)$ Let $m \leq k t$ and $k>2$.

Then $t=0$ implies $m=0$, done in part 2, so by the reduction (1), $t=1$ or $m \leq k$. But $t=1$ and $m \leq k t$ means $m \leq k$, either way.

$A$ further reduction. Suppose $t>1$. Write $G$ as $[0, t)$ or as $(0, t)+I_{2}+\cdots+I_{m}$ depending on whether $G$ is unicyclic or not. Map each $[n, n+1) k$-to-1 onto the $n$th open interval of $H$ using Lemma 5 , for $n=1,2, \ldots, t-1$. This leaves only one open interval in $H$ not mapped onto and $G$ is essentially the same, $[0,1)$ or $(0,1)+I_{2}+\cdots+I_{m}$. In the former case use Lemma 5 to map $[0,1)$ onto the rest of $H$, and in the latter case map the $m$ intervals of $G$ onto $(0,1)$ using Lemma 3.

$(\rightarrow)$ Let $m>k t, k>2$.

Since $m>k t, G$ is not unicyclic. Also, $m>k t$ plus the reduction (1) $m \leq k t$ or $t \leq 1$ implies $t \leq 1$. If $t=0$, Lemma 2 is used, and if $t=1$, Lemma 4 is used.

4. Proof of (B).

$(\leftarrow)$ Suppose $m=2 t$ and $k=2$.

Since $m=2 t$ and the $m=t=0$ case was done in part 2, we assume that $G$ can be rearranged as $2 t$ open intervals and $H$ as $t$ open intervals. But then $G$ obviously maps 2-to-1 onto $H$.

$(\rightarrow)$ Suppose $m \neq 2 t$ and $k=2$.

$$
\begin{aligned}
& \text { If } t=0 \text {, Lemma } 2 \text { is used, since } m \neq 0 . \\
& \text { If } m=0 \text {, Lemma } 1 \text { is used, since } t \neq 0 .
\end{aligned}
$$

The case $m=1$ is covered by reference fact 1 .

Hence, by the reduction (1) we can assume that $t=1$ or $m=2$.

If $t=1$, then $m \neq 2$ since $m \neq 2 t$, and $m>2$ is covered by Lemma 4 .

If $t>1$ and $m=2$, then two new points can be added to $G$ and rearranged as $[0,1)$, and $H$ with one new point rearranges as $t-1$ open intervals. This case is covered by Lemma 1 .

This completes the proof of Theorem 1 . 
THEOREM 2. Suppose $G$ and $H$ are finite graphs, one of them is acyclic, and $k>1$. Then

(1) If $H$ is acyclic, then there is no $k$-to-1 function from $G$ onto $H$ with only finitely many discontinuities.

(2) If $G$ is acyclic and $H$ is any multicyclic graph, then there is a $k$-to-1 function from $G$ onto $H$ with only finitely many discontinuities iff $k>2$.

ProOF. 1. If $H$ is an acyclic graph then $H$ rearranges into $[0,1]$ and there is no $k$-to-1 $F D$-function from any graph $G$ onto $H$ by reference fact 2 .

2. If $k=2$, and $G$ is acyclic, then there is no 2-to-1 $F D$-function defined on $G$ at all, by reference fact 1 .

3. If $k>2, G$ is acyclic, and $H$ is unicyclic, then there is a $k$-to- $1 F D$-function from $[0,1]$ onto $[0,1)$ by reference fact 6 .

4. If $k>2, G$ is acyclic and $H$ is $(t+1)$-multicyclic with $t>0$, then $H$ can be rearranged as $J_{1}+\cdots+J_{t}$, open intervals, and $G$ as $[0, t]$. There is a $k$-to- 1 function (from reference fact 5) from $[0,1]$ onto $J_{1}$ and for each $i=2,3, \ldots, t$, there is a $k$-to-1 function (from Lemma 5 ) from $(i-1, i]$ onto $J_{i}$. The union of these functions is a $k$-to-1 $F D$-function from $G$ onto $H$.

ADDENDUM. The answer to Question 1 can be found in [14].

\section{REFERENCES}

1. K. Borsuk and R. Molski, On a class of continuous maps, Fund. Math. 45 (1958), 84-98.

2. P. Civin, Two-to-one mappings of manifolds, Duke Math. J. 10 (1943), 49-57.

3. P. Gilbert, n-to-one mappings of linear graphs, Duke Math. J. 9 (1942), 475-486.

4. W. H. Gottschalk, On k-to-1 transformations, Bull. Amer. Math. Soc. 53 (1947), 168-169.

5. O. G. Harrold, The non-existence of a certain type of continuous transformation, Duke Math. J. 5 (1939), 789-793.

6. __ Exactly $(k, 1)$ transformations on connected linear graphs, Amer. J. Math. 62 (1940), 823-834.

7. J. W. Heath, Every exactly 2-to-1 function on the reals has an infinite number of discontinuities, Proc. Amer. Math. Soc. 98 (1986), 369-373.

8. $\_$- $k$-to-1 functions on arcs for $k$ even, Proc. Amer. Math. Soc. 101 (1987), 387-391.

9. only finitely many discontinuities, Trans. Amer. Math. Soc. 316 (1988), 293-305.

10. H. Katsuura and K. Kellum, k-to-1 functions on an arc, Proc. Amer. Math. Soc. 101 (1987), $629-633$.

11. H. Katsuura, $k$-to-1 functions on $(0,1)$, preprint.

12. R. Levy, How to go around in circles, preprint.

13. V. Martin and J. H. Roberts, Two-to-one transformations on 2-manifolds, Trans. Amer. Math. Soc. 49 (1941), 1-17.

14. J. Mioduszewski, On two-to-one continuous functions, Dissertationes Math. (Rozprawy Mat.) 24 (1961), 42.

15. S. B. Nadler, Jr. and L. E. Ward, Jr., Concerning exactly $(n, 1)$ images of continua, Proc. Amer. Math. Soc. 87 (1983), 351-354.

16. J. H. Roberts, Two-to-one transformations, Duke Math. J. 6 (1940), 256-262.

Department of Mathematics, Fat, Auburn University, Auburn, Alabama 36849-5310 land

Current address: Department of Mathematics, University of Reading, Reading RG6 2AX, Eng- 